\title{
Research on Some Topology Model of Wireless Sensor Networks Based on Complex Network Theory
}

\author{
LU Kui ${ }^{1}$, JIN Xin ${ }^{1}$, ZHAO Jian-dong ${ }^{1}$,SUN Jian-peng ${ }^{2}$ \\ 1.College of Computer Science and Engineering, Anhui University of Science and \\ Technology,Huainan 232001, China; 2.College of Electrical and Information Engineering, Anhui \\ University of Science and Technology,Huainan 232001,China
}

Keywords: Wireless sensor network (WSN), a complex network, small-world networks and scale-free feature

\begin{abstract}
The placement of the wireless sensor networks in the wild or the unpopulated area makes it difficult for energy supplement, and vulnerable to damage, so it is of great importance to improve the overall network energy efficiency and prolong the maximum life cycle. In consideration of the energy consumption existed in the wireless sensor networks, a wireless sensor networks model featured by small world effect is put forward in the paper by introducing hyperlink through the addition of super nodes to the wireless sensor networks, based on the characteristics of the complex small-world networks. From the perspective of complex network, the influence of adding super nodes to the sensor networks on the energy efficiency is analyzed. Simulation studies show that the proper addition of super nodes to the wireless sensor networks exerts a clear improvement on network transmission and energy efficiency.
\end{abstract}

\section{Introduction}

Wireless sensor is a distributed self-organization networks composed of a large quantity of ubiquitous micro sensor nodes with wireless communication and computing capabilities, which has been widely applied to the fields: disaster rescue, medical rescue, forest fire alarm, volcano detection, environmental monitoring and military.

Small-world phenomenon(small-world effect) is also known as Six Degrees of Separation, which is characterized as small average path length and large clustering coefficient.

Based on the characteristics of complex small-world networks, the paper introduces a super node with higher energy, storage capacity and data processing capacity, which makes up a super link that is a reliable way to communicate with the sink node, and puts forwards a network model based on small world effect in wireless sensor networks. Still, in consideration of the parameters, like: the average path length, the change rate of the average path length and the network energy saving ratio, the paper makes a simulation analysis and test of the appropriate addition of the super nodes to construct the super link that makes the wireless sensor networks have the the following characteristics: the small world effect, the reduced data transmission delay and the improved energy efficiency.

\section{Background}

Small world phenomenon is often seen in many real networks, such as the Internet, scientist cooperation networks, social relation networks and WWW network, etc., and is also widely used in the field of $\mathrm{P} 2 \mathrm{P}$ systems and neural networks ${ }^{[1-2]}$. Real networks can be divided into two categories. One is the relation network, in which the distance between the nodes is not dependent on the network topology and node location, but on the calculation of hop count. The other one is the spatial network, in which the connection between the nodes is closely related to the distance between the nodes. The usual study on complex networks belongs to the study of relation schema in which relation networks are taken from the social relations or the technical networks, while wireless networks and wireless sensor networks, due to the limitation of its transmission radius, is the spatial network, which belongs to the study of space map. The topological structure of spatial network is closely related to the connectivity and transmission radius. Recent studies have indicated that "small 
world characteristics" also exists in some heteroideous wireless sensor networks.

The main indicator to describe small-world networks is characteristic path length and clustering coefficient, which are also important factors affecting the performance of network topology in wireless sensor networks. In the wireless sensor networks, multi hop is used to transmit data, in which the shorter path length indicates a fewer communication hops needed to transmit data between nodes, which helps to reduce the communication overhead and the energy consumption of sensor nodes. Clustering coefficient shows the degree of aggregation of nodes in the network. Large clustering coefficient can improve the robustness of the nodes in the whole networks as well as the fault tolerance ${ }^{[3]}$. Therefore, it is of great realistic significance to construct a networks with small characteristic path length and high clustering coefficient, a wireless sensor networks featured by small world.

Recently, studies have indicated that the construction of wireless sensor networks with small world effect plays a very important role in reducing the communication cost of the whole networks, improving the efficiency of data transmission, reducing the average energy consumption of each node and prolonging the survival time of the networks. In 2003, Helmy, through random link reconnection and bordered experiment, verified the small world phenomenon in the wireless sensor networks, which shows that the addition of shortcuts in the sensor networks can lead to a decreased average path length of the network nodes. In the same year, he also designed a network resource search mechanism based on the small world effect, which effectively reduces the path between the data query source and the target node, and reduces the communication overhead. Adding several wired long-range links to the wireless sensor networks, Sharma constructed the Hybrib heterogeneous sensor networks, which also shows the property of small world. The result shows that these connections reduce the average hops of the networks resulting in less energy consumption not only for single node but also for the whole networks.

Cavalcanti adds even-distributed special heteroideous nodes with longer communication distance to the ad hoc networks. According to the Percolation theory, these special nodes with two different transmission powers can improve the connectivity of the whole networks and reduce the average path length. In 2010, Guidoni studied how to construct a small world effect in the wireless sensor networks by adding directed shortcuts. Jiang and Chen bordered wireless sensor networks through the mobile nodes, rendering networks with the property of small world. Nardis designed a small world network topology which is suitable for ad hoc networks with limited energy, and further designed a small world network router ${ }^{[4]}$.

\section{Sensor network model for small world effect}

In the large-scale application of the wireless sensor networks with large space and long time, the key problem in the application of sensor networks is the limitation of the node resources, especially energy resources. Therefore, in the design of the wireless sensor network topology, priority should be given to how to achieve energy efficiency in order to prolong the network lifetime. The construction of topology is based on the deployment of the nodes.

3.1 The design of network model
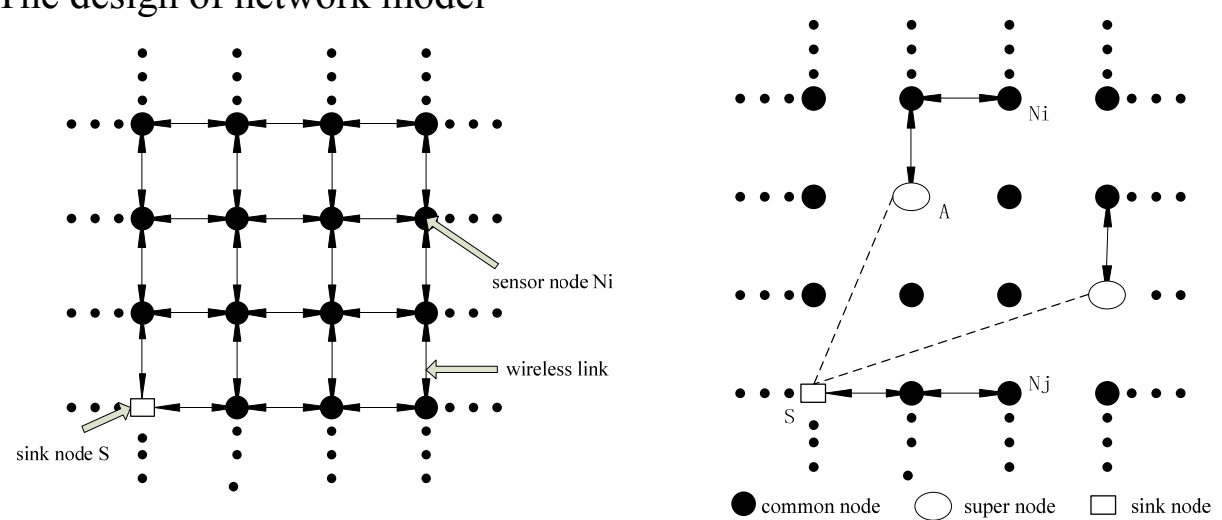

Fig.1 The connectivity graph of the WSN deployed with a uniform distribution Fig.2 Network communication after adding super nodes 
This paper is about how to build a network topology with small world effect in the deployment of the nodes in the mesh structure. Sensor networks are usually used to fix the relative position of the sensor nodes, and the networks need a long-term monitoring. The network model used in this paper is $\mathrm{N}$ sensor nodes deployed evenly in $\mathrm{Z}$ of a $\mathrm{X}^{*} \mathrm{Y}$ rectangular area, with each node evenly distributed at the crossing point. In order to avoid the changes of network topology, all of the sensor nodes are supposed to be static once deployed. And these nodes are homogeneous with the same energy and the same communication radius, and their position is known. The position is marked as the plane coordinates $(\mathrm{x}, \mathrm{y})$, among which $0 \leq \mathrm{x} \leq \mathrm{X}-1,0 \leq \mathrm{y} \leq \mathrm{Y}-1$. The network topology, as shown in Figure 1, forms a Mesh structure. The sensor nodes transmit and receive data only with their adjacent nodes in vertical and horizontal position, while the sink node can be located anywhere in the monitoring area. The sensor nodes transmit data to the sink node at a certain rate, and the sink node can be connected with other cable networks or wireless network, and finally to the user terminal.

Suppose that the greedy routing strategy is applied to the sensor node, that is, the data is transmitted to the sink node by the shortest distance. When the node Ni needs to send data to the sink node $\mathrm{S}$, it first finds the node closest to the sink node in the neighboring node, and then forwards the data to the neighbor node. The neighbor node and the subsequent node are followed by a repeat of the forward process until the data packet reaches the sink node. If a node that receives a data packet finds that there is no shorter distance to the sink node in its neighbor node, the node will discard the data. The algorithm can ensure that all nodes transmit data to the sink node with the least number of hops.

In order to construct a network model of small world effect in the environment of the heteroideous wireless sensor network, a super node with higher energy, storage capacity and data processing capability is introduced. These super nodes make it possible for the long-distance communication without the limitation of broadband, and form some super links which are reliable shortcuts for the direct communication between the super nodes and the sink nodes, or for jumping to the sink nodes through other super nodes. Super node is required to have adequate energy and adequate communication radius. Besides, super nodes should be able to communicate not only with the sink node and super node directly, but also with the common node. So the super node must have two kinds of transmitting frequency, one is for the communication with the sink node or the super node, the other for the communication with common node. Assuming that the super nodes are equally distributed in the monitoring environment and broadcast their position in the networks, then the common nodes can get the location of the super nodes, so the greedy routing strategy is still used in the introduction of the "shortcut" in the network. According to the location broadcasted by the super node, nodes in the network choose the shortest path length to the sink node, and select the shortest as its own "shortcut". In the transmission of data, the node uses the greedy routing algorithm to find if there is shorter communication hops by comparing "shortcuts". If it is, then select the "shortcut" to send data to the sink node; otherwise, use the original wireless multi hop link to send data.

Suppose that the plane coordinate position of the common node Ni is $\left(\mathrm{x}_{\mathrm{i}}, \mathrm{y}_{\mathrm{i}}\right)$, the position of the common node $\mathrm{Nj}$ is $\left(\mathrm{x}_{\mathrm{j}}, \mathrm{y}_{\mathrm{j}}\right)$, and that of sink node Sink is $\left(\mathrm{x}_{\mathrm{s}}, \mathrm{y}_{\mathrm{s}}\right)$. Firstly calculate the shortest hop count distance for common node $\mathrm{N}_{\mathrm{j}}$ and $\mathrm{N}_{\mathrm{j}}$ to send data to the sink node without passing through the super node:

$$
\begin{aligned}
& H\left(N_{i}, S\right)=\left|x_{i}-x_{s}\right|+\left|y_{i}-y_{s}\right| \\
& H\left(N_{j}, S\right)=\left|x_{j}-x_{s}\right|+\left|y_{j}-y_{s}\right|
\end{aligned}
$$

As shown in Figure 2, if the common node $\mathrm{Ni}$ is far away from the sink node, Ni firstly sends data to the nearest super node in a multi hop way, and then directly to the sink node through the super link. At this time, the shortest hop count for node Ni to sent data to the nearest super node is:

$$
H^{\prime}\left(N_{i}, S\right)=\left|x_{i}-x_{A}\right|+\left|y_{i}-y_{A}\right|
$$


Since the super node and the sink node are not short of energy, it is unnecessary to consider the energy consumption of the super node and the super link. When $H\left(N_{i}, S\right) \geq H^{\prime}\left(N_{j}, S\right)$, the node chooses super node to send data. On the contrary, when the common node $\mathrm{Nj}$ is closer to the sink node, when $H\left(N_{i}, S\right)<H^{\prime}\left(N_{j}, S\right)$, the node $\mathrm{Nj}$ does not select the super node to send data, which reaches the sink node through the common node by wireless multi hop.

3.2 Simulation analysis of small world effect

Small world network has a smaller characteristic path length. In a less complex networks, the characteristic path length refers to the average value of the shortest path between any two points in the networks. In the wireless sensor networks, data from the sensor node gather at the sink node. Since the transmission of data is clearly directed, data flow follows the many-to-one convergence mode. In the simulation analysis, a specific location is set for the sink node, so the average path length needs to be modified according to the special characteristics of the sensor network. The average path length is the average value of the communication hops that are needed by all of the nodes to send data to the super node or the sink node in a sensor networks, shown as:

$$
A P L=\frac{1}{n} \sum_{i=1}^{n} H_{i-\sin k}
$$

Of which, $\mathrm{n}$ stands for the quantity of sensor nodes in the networks, $H_{i \text {-sink }}$ stands for the communication hop distance for node $\mathrm{i}$ from the super node to the sink node, that is, the hop count of the shortest path of the common node i to the sink node in the sensor networks, shown as:

$$
H_{i-\sin k}=\min \left(H\left(N_{i}, S\right), H^{\prime}\left(N_{i}, S\right)\right)
$$

Supposing adding $\mu$ super nodes to a homogeneous network, the probability of the common node transmitting data through a super node is $\rho_{i, u}$, in the application of a large-scale wireless sensor network, when $\rho_{i, u}$ increases as the value of $\mu$ increases, the average path length of the network node to the super node or to the sink node is about:

$$
\operatorname{APL}(\mu)=\frac{1}{n} \sum_{i=1}^{n}\left[\rho_{t, \mu} H^{\prime}\left(N_{i}, s\right)+\left(1-\rho_{i, \mu}\right) H\left(N_{i}, s\right)\right]
$$

\section{$(1-6)$}

In the initial homogeneous network, when there is no increase in the amount of the super node, the average path length of the network node to the sink node is:

$$
\operatorname{APL}(0)=\frac{1}{n} \sum_{i=1}^{n} H\left(N_{i}, s\right)
$$

So, between the homogeneous mesh networks and the networks generated from the super links constructed by adding supper nodes, the ratio of the average path length of the node to the super node or sink node is defined as the change rate of the average path length, shown as:

$$
\operatorname{APLR}(\mu)=\frac{A P L(\mu)}{\operatorname{APL}(0)}
$$

\section{(1-8)}

In the sensor networks, the energy consumption of the nodes is mainly used for data processing and transmission, so the network energy consumption is closely related to the average path length of data transmission. Energy saving ratio is defined as:

$$
E S R(\mu)=1-\frac{A P L(\mu)}{A P L(0)}
$$

The greater the energy saving ratio, the higher the energy efficiency of the sensor network.

When the super node is added to the wireless sensor network, the common node around the super node transmits data through a super node. When the network topology changes, network clustering feature arises, which is considered to be of a higher clustering coefficient. 
In the simulation experiment, a $20 * 20$ Mesh networks is applied, with sink node deployed at $(0,0)$ and at the center of the networks $(10,10)$, and common node deployed at the crossing point in the Mesh networks, supposing the super node are deployed evenly in the monitoring environment, shown as figure 4:

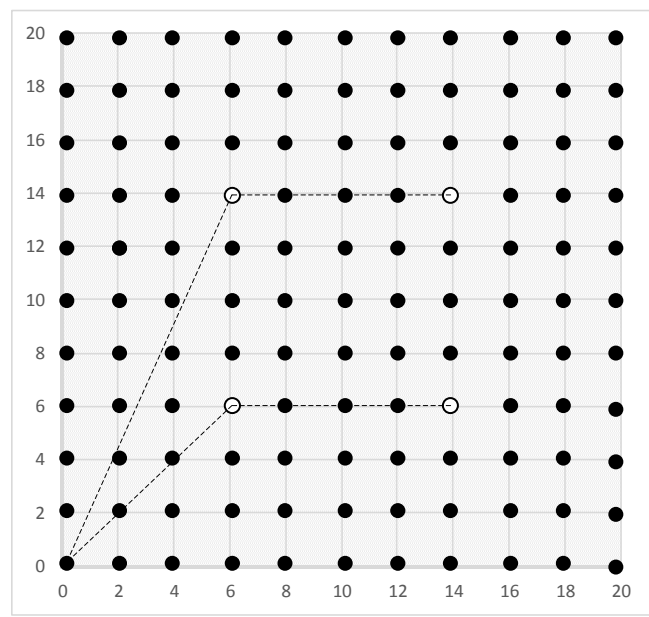

Fig.4 Deployment of network nodes

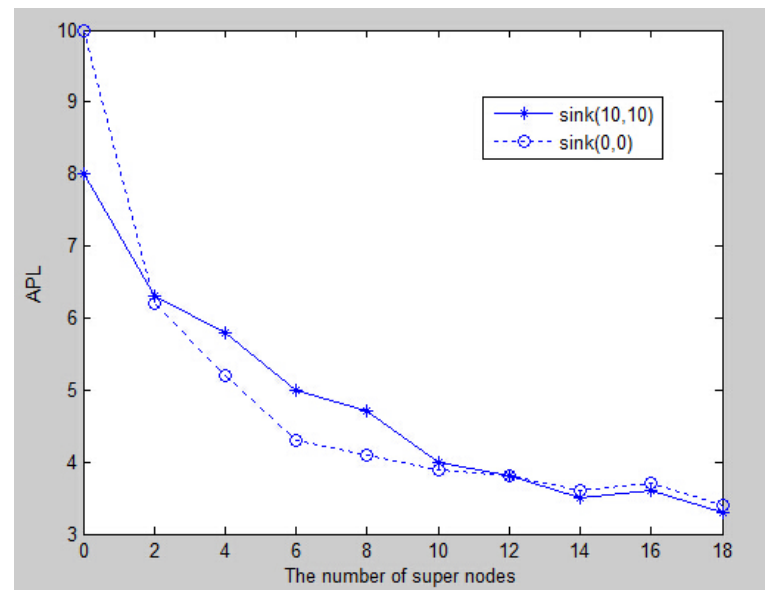

Fig.5 Average Path Length(APL) with the different number of super nodes

In Figure 5, the relationship between the number of super nodes added in the homogeneous Mesh networks and the average path length APL $(\mu)$ is given. By Figure 5, the average path length of the network nodes to the super sink node decreases rapidly after the addition of a small amount of super nodes in the network, especially when the position of the sink node is at $(0,0)$ instead of $(10,10)$, the improvement is more obvious. When the position of the sink node is at $(0,0)$, and the number of super nodes added in the homogeneous networks is 1-6, the average path length decrease from 10 to 4 . However, when the super node continues to increase, the average path length decreases very slowly, which indicates that only a small amount of super nodes added in the homogeneous network, the small world effect with small average path length can be constructed.

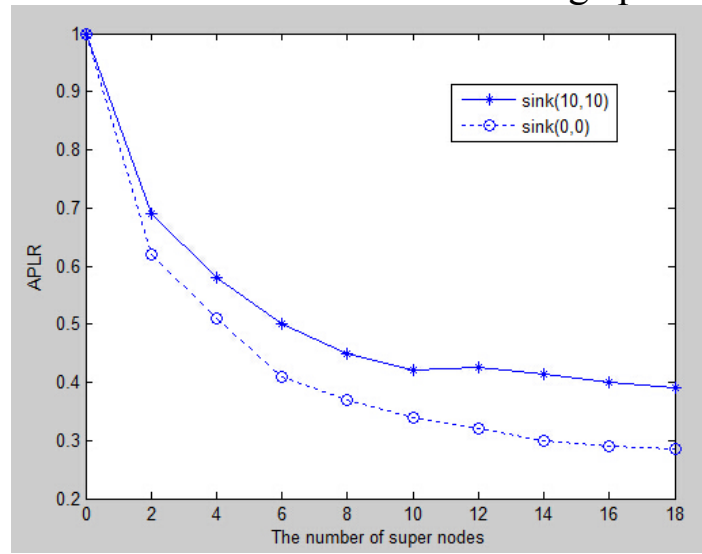

Fig.6 Average path length reduction ratio with the different number of super nodes 


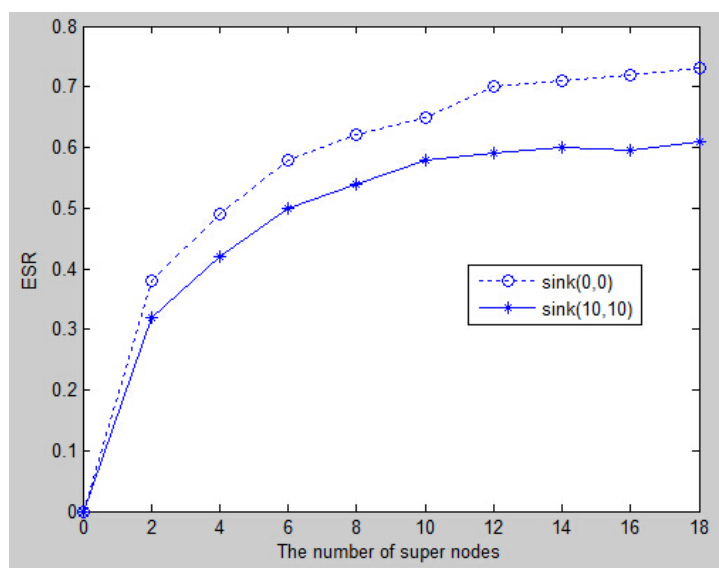

Fig.7 Node energy save ratio with the different number of super nodes

Figure 6 shows the relationship between change rate APLR (MU) of the average path length and the addition of super nodes. By Figure 6, in the two simulation environment, only with 6 super nodes added, the average path length of the network will be reduced to about $45 \%$, but the reduction is so obvious as more super nodes are added. So, the reasonable addition of the super nodes will not greatly affect the topology of the network, but the network path length of the transmission of data will be significantly reduced, which is the embodiment of complex networks with the small world effect.

Figure 7 shows the relationship between the network energy saving ratio and the addition of super node. It can be found that the network energy saving ratio can reach about $50 \%$ when the super link is increased from one to six, but the effect is not obvious after the extra addition of the super link. When the sink node is located at $(0,0)$, the energy saving effect can be significantly improved compared to that in $(10,10)$.

\section{Conclusion}

This paper first introduces the origin and development history of small world network, and then analyzes the application and research of small world effect in wireless sensor networks. Based on the characteristics of complex small-world networks, the paper introduces a super node with higher energy, storage capacity and data processing capacity, which makes up a super link that is a reliable way to communicate with the sink node, and puts forwards a network model based on small world effect in wireless sensor networks. Still, in consideration of the parameters, like:the average path length, the change rate of the average path length and the network energy saving ratio, the paper makes a simulation analysis and test of the appropriate addition of super nodes to construct the super link that makes the wireless sensor networks have the the following characteristics: the small world effect, the reduced data transmission delay and the improved energy efficiency.

In this paper, the even distribution of the nodes is studied, which can introduce the super node and the super link, and then construct the sensor network topology with small world effect. Such Problems as: the random uneven distribution of the nodes, how to optimize the deployment of the super nodes, and the further application of the small world effect in the sensor networks, need to be further studied.

\section{References}

[1]Xiucai ye, Li Xu, Liwei Lin. Small-word Model Based Topology Optimization in Wireless Sensor Networks. Proceeding of the IEEE International Conference on Informat ion Science and Engineering.2008,12:102-106

[2]Ken Y.K Hui,Johe C.S Lui,David K.Y. Yau. Small-world overlay P2P networks construction,management and handling of dynamic flash crowds, Computer Networks.2006,50(15):2727-2746

[3]Pengsheng Zheng,Wansheng Tang,Jiangxiong Zhang.A simple method for designing efficient 
small-world neural networks.Neural Networks.2010,23(2):155-159

[4]L. D. Narids, M. G. D. Benedetto, The Small Worlad Routing Strategy:A methodological framework for driving the emerging topology of energy-constrained multi-hop wireless networks. Proceedings of the 2006 IEEE Radio and Wireless Simposiu,.2006:599-602

[5] Geng zhong Zheng, Sanyang Liu, Xiaogang Qi. Scale-free topology evolution for wireless sensor networks with reconstruction mechanism. Computers and Electrical Engineering, 2012 (38): 643-651

[6] TONG C, NIU J W, QU G Z, et al. Complex networks properties analysis for mobile Ad Hoc networks. IET Communications, 2012, 6(4):370-380

[7] Xiao juan Luo, Huiqun Yu , Constructing Wireless Sensor Network Model Based on Small World Concept, 2010 3rd International Conference on Advanced Computer Theory and Engineer ing, Proceedings, 2010(5):V5501-V5505

[8] Nan Jiang, Huan Chen, and Xiang Xiao. A Local World Evolving Model for Energy- Constrai ned Wireless Sensor Networks. International Journal of Distributed Sensor Networks. Article ID:542389, 9 pages, 2012

[9] S. D. Li, L. X. Li, Y. X. Y, A local-world heterogeneous model of wireless sensor networks with node and link diversity. Physica A. 2011， 390: 1182-119

[10]Wang Xiao-fan, Li Xiang \& Chen Guan-rong, Introduction to Network Science, [M], Higher Education Press, 2012

[11]Zheng Geng-zhong, Liu San-yang \& Qi Xiao-gang, Overview on Topology of Wireless Sensor Networks Based on Small World Network Model, [J], Control and Decision Making, 2010,25(12):1761-1768.

[12]Zhu Bao-hong, Research on topology control of Wireless Sensor Networks, [D], Yanshan University, 2012

[13]Zeng Yuan, Research on the Application of Complex Network Theory in WSNs, [D], Nanjing Posts and Telecommunications University, 2012

[14]Luo Xiao-juan,Yu Hui-qun \& Leng Chun-xia, Local world dynamic evolution model based on wireless sensor network [J] Journal of East China University of Science and Engeneering, Natural Science Edition, ,2012,38(002):2016-220

[15]Wang J, Fan J, Wang Y. A novel local nonlinear preferential attachment model for Wireless Sensor Networks[C]// Computer Science and Network Technology (ICCSNT), 2012 2nd International Conference onIEEE, 2012:40-44.

[16]Faragó A. Network Topology Models for Multihop Wireless Networks[J]. Isrn Communications \& Networking, 2012, 2012(2012). 